\title{
émulations
}

\section{Maurice Godelier - Suivre Jésus et faire du business. Une petite société tribale dans la mondialisation}

Christophe Premat

Émulations - Revue de sciences sociales

2019. Comptes rendus critiques. En ligne

Article disponible à l'adresse suivante

https://ojs.uclouvain.be/index.php/emulations/article/view/7573

\section{Pour citer cet article}

Christophe Premat, « Maurice Godelier - Suivre Jésus et faire du business. Une petite société tribale dans la mondialisation ", Émulations, en ligne. Mise en ligne le 18 mars 2019.

DOI : 10.14428/emulations.cr.054

Distribution électronique : Université catholique de Louvain (Belgique) : ojs.uclouvain.be

(C) Cet article est mis à disposition selon les termes de la Licence Creative Commons Attribution, Pas d'Utilisation Commerciale 4.0 International. http://creativecommons.org/licenses/by-nc/4.0/

Éditeur : Émulations - Revue de sciences sociales / Presses universitaires de Louvain https://ojs.uclouvain.be/index.php/emulations

ISSN électronique : 1784-5734

UCL PRESSES

UNIVERSITAIRES 


\title{
Maurice Godelier - Suivre Jésus et faire du business. Une petite société tribale dans la mondialisation
}

\author{
Christophe Premat $^{1}$
}

Recensé : Maurice Godelier (2017), Suivre Jésus et faire du business. Une petite société tribale dans la mondialisation, Vincennes, Thierry Marchaisse, $170 \mathrm{p}$.

«[Les humains] produisent de nouvelles formes d'existence sociale, donc de sociétés, pour continuer à vivre. Et en transformant leurs manières de vivre, ils transforment leurs manières de penser et d'agir, donc leur culture », écrivait Maurice Godelier (2009 : 10) lorsqu'il analysait les relations entre communauté, société, culture et identité. En effet, une culture n'est jamais statique, mais s'appréhende dans sa dynamique évolutive (Verbunt, 2012), c'est-à-dire que les êtres humains modifient en permanence les règles de vie commune pour pouvoir continuer la «vie sociale comme telle» (Castoriadis, 1986 : 165). Godelier énonce ici les caractéristiques générales d'une culture à partir de ses enquêtes de terrain et de ses analyses spécifiques du fonctionnement social des Baruya de Nouvelle-Guinée. En l'occurrence, dans son dernier ouvrage paru en 2017 aux éditions Thierry Marchaisse, Godelier rend compte de l'évolution de cette tribu qu'il a appris à connaitre à la fin des années 1960. La tribu est l'organisation sociale et politique fondée sur des relations de parenté (Ben Hounet, 2009), elle est la cellule décisionnelle en matière d'allocation des ressources à ses membres (Godelier, 1977). L'ouvrage oscille entre une analyse des souvenirs de l'auteur, une synthèse des différents travaux sur les Baruya et une réflexion méthodologique sur les manières de produire des analyses anthropologiques rigoureuses. L'ouvrage comporte trois parties : en premier lieu un rappel des recherches de l'auteur sur la trahison du secret des Baruya (Godelier, 1988), puis une analyse des outils méthodologiques utilisés par les anthropologues et enfin une réflexion sur l'évolution des Baruya au contact des Églises luthériennes. Son originalité tient au fait qu'il se situe entre une synthèse de travaux antérieurs de l'auteur depuis 1969 et un suivi de l'évolution des Baruya. Le livre contient d'ailleurs des photographies issues des terrains d'investigation avec notamment les souvenirs des premiers informateurs de ces tribus et l'arrivée des premières Églises luthériennes au début des années 2010 (Godelier, 2017 : 98). Depuis 1969, Godelier avait en effet consacré une vingtaine de publications majeures sur les Baruya portant sur les outils, les ressources, le pouvoir et la temporalité propres à cette tribu. C'est en travaillant sur les Baruya que Godelier

${ }^{1}$ Maître de conférences en études culturelles au département d'études romanes et classiques de I'Université de Stockholm. 
avait publié l'un de ses ouvrages majeurs en 1982, La Production des Grands Hommes (Godelier, 1982).

L'existence des Baruya a été révélée en 1951, date à laquelle les influences extérieures ont inévitablement altéré les rituels et les comportements de ces tribus qui étaient déjà en rivalité avec d'autres tribus voisines (Godelier, 2017 : 24). Les Baruya appartenaient en effet à l'ensemble culturel Anga, ce qui correspondait au début des années 1980 à une population de 65000 personnes; les Baruya comptaient environ 2500 personnes et habitaient dans deux vallées proches des côtes du golfe de Papouasie (Lory, 1982 : 248). Les Baruya possédaient un secret savamment entretenu dans la multiplication de rites initiatiques consacrant la domination masculine. L'auteur décrit la manière dont ces rites sont conçus à partir de la construction d'une «maison cérémonielle», la tsimia (Godelier, 2017 : 25). Quand les garçons entraient à l'âge adulte, ils extériorisaient leur part féminine pour se transformer en hommes (Godelier, 1985 : 161), certains devenant de «grands hommes » susceptibles de diriger la tribu (Godelier, 2017 : 33). La tsimia est perçue comme un corps représentant l'ensemble des jeunes Baruya appelés à exercer une fonction au sein de la tribu (Godelier, $2017: 26$ ).

Les rites manifestent une solidarité masculine qui fait tenir ensemble la tribu Baruya et pourtant le cercle des trahisons a émergé à partir d'un mythe ancien, celui d'un orphelin qui, faute d'avoir été aidé par les autres initiés, est allé chercher l'assistance de villages voisins enclenchant de facto un cycle de vengeances, d'échanges de femmes et une redistribution des relations de pouvoir entre les différents villages (Godelier, 2017 : 36). Cette trahison a souvent tourné à l'avantage des Baruya eux-mêmes, dans la négociation des échanges de terres et la construction des interlignages (Godelier, 2017 : 45). La trahison a été selon Godelier le fondement de la croissance de la tribu des Baruya, elle a constitué un acte politique fort, car elle a activé les métissages interlignagiers et a même eu une influence sur l'évolution des langues locales et des coutumes (Godelier, 2017 : 37). La trahison révèle plutôt un mouvement de décomposition des solidarités villageoises (Godelier, 2017 : 44). Les mariages par alliances sont le fruit de cette trahison qui semble renvoyer métonymiquement à un processus de métissage inévitable (Nathan, 1993 : 12). Les alliances matrimoniales permettent aux Baruya d'acquérir de nouveaux territoires et d'avoir une régulation des rapports sociaux par le biais des beaux-frères qui deviennent plus importants que les frères (Godelier, $2017: 47$ ).

La description de ce système de parenté montre comment les échanges régulent d'une certaine manière les relations et le métissage avec d'autres lignées villageoises. Dans les trahisons, certains Baruya peuvent devenir des réfugiés et être accueillis en hôtes dans une tribu voisine avec cependant la perte de l'identité qui avait été conférée par les rites d'initiation originaux. Godelier poursuit l'analyse des trahisons avec parfois la révélation d'alliances paradoxales. En effet, les Baruya avaient du respect pour leurs grands guerriers, les aoulatta, qui se battaient contre les grands guerriers d'autres tribus voisines (Godelier, 2017 : 51). Selon l'auteur, certains aoulatta pouvaient abuser de leur supériorité ce qui conduisait certains villages à chercher l'appui d'un ennemi voisin pour se débarrasser du guerrier encombrant (Godelier, 2017 : 52). Il existe comme un mécanisme de régulation des conflits et des trahisons successives. L'auteur 
rapporte d'autres analyses innovantes qu'il fit en 1988 à partir de mythes permettant de comprendre la manière dont les hommes ont essayé de dérober le pouvoir des femmes sur la vie et la reproduction. Les femmes sont associées dans les mythes Baruya au pouvoir créateur et à la maitrise des forces vitales, d'où l'importance pour les jeunes garçons d'être initiés pour les dominer (Godelier, 2017 : 56).

L'ouvrage aborde ensuite la question des outils méthodologiques à la disposition des anthropologues qui doivent d'abord construire le lieu de leur observation, nouer une relation de complicité avec des informateurs de la culture observée pour pouvoir commencer leurs recherches (Godelier, 2017 : 64). Il faut rappeler au lecteur que les années 1960 furent marquées par l'utilisation du film comme moyen d'investigation ethnographique avec en particulier la captation de rituels. Godelier rappelle qu'à l'époque il était difficile d'avoir des fonds de recherche pour utiliser ces techniques tant la concurrence était forte notamment avec les documentaires de Jean Rouch (Godelier, 2017 : 77). En réalité, la caméra est un objet technique doté du pouvoir de faire revivre ces rituels pour les soumettre à l'analyse, elle implique un dispositif négocié de relations entre la culture observée acceptant cette intrusion et l'équipe chargée d'enregistrer les séquences. La photographie ne permettait pas de rendre véritablement compte du mouvement entre les personnes et les objets; en revanche, il est dommage selon nous que l'ouvrage n'évoque pas davantage les différentes manières d'enquêter sur ces rites.

Godelier met en évidence le fait que l'anthropologue devient aussi dans ce cadre l'entremetteur susceptible de faire accepter le projet comme ce fut le cas avec la demande formulée par Marek et Allison Jablonko de filmer les rites des Baruya (Godelier, 2017 : 66). La caméra conduit l'anthropologue à prendre des risques et à transgresser des tabous des Baruya sur les rituels féminins/masculins (Godelier, 2017 : 72), elle fonctionne alors comme médiation culturelle (Godelier, 2017 : 71). Dans l'enregistrement sonore, il y a chez Godelier une volonté de filmer une observation participante dans laquelle la voix de l'anthropologue se mêle à d'autres voix. L'anthropologue se décentre ainsi de ce qu'il étudie à partir d'un espace polyphone (Piault, 2013). En revanche, une réflexion sur l'évolution des techniques d'enregistrement des rituels (caméscope, caméra mobile et aujourd'hui les webcams et les téléphones mobiles) est absente de l'ouvrage, alors même que l'auteur s'interroge sur ce que l'anthropologie peut apporter au champ des sciences sociales (Godelier, 2017 : 74). Godelier évoque par la suite la manière dont il avait répondu à une sollicitation de Ian Dunlop ${ }^{2}$ de filmer les Baruya à partir d'un échange de barres de sel contre des capes d'écorce et des flèches avec les Youndouyé, une tribu voisine des Baruya (Godelier, 2017 : 78). L'interaction entre le cinéaste et l'anthropologue qui devient le véritable metteur en scène de la relation avec les Baruya est remarquable, elle offre de surcroit une archive concrète permettant de revenir sur une forme originale d'observation participante (Godelier, 2017 : 85). Certes, il y a les photographies, mais le cahier d'images laissé par Godelier (Godelier, 2017 : 89-104) ne rend pas suffisamment compte de la dynamique des interactions, contrairement à la caméra.

${ }^{2}$ Le film de Ian Dunlop tiré de ces séquences s'intitule Towards Baruya Manhood et est sorti en 1972. 
Ces premières enquêtes filmées permettent à l'auteur d'évaluer les changements de comportement des Baruya et de leurs voisins suite à leurs premiers contacts avec les colons australiens en 1951. Les analyses de la fin de l'ouvrage relèvent de l'ethnographie d'un processus de colonisation revêtant trois étapes : la christianisation, la scolarisation et l'apprentissage des codes de la mondialisation des échanges. La territorialisation de ces communautés s'est accompagnée d'un processus de représentation politique avec des consol, c'est-à-dire des chefs professionnels recevant un salaire en échange d'un règlement des conflits locaux (Godelier, 2017 : 125). La christianisation a été marquée de son côté par l'arrivée d'Églises luthériennes concurrentes tolérant certaines coutumes locales des Baruya en échange d'une pratique de la foi (Godelier, 2017 : 128). Les premiers missionnaires dans les années 1960 avaient eu du mal à convertir les Baruya parce qu'ils voulaient éliminer la plupart des rituels de cette tribu (Godelier, 2017 : 118). Godelier rappelle que ces Églises se sont adaptées au cours du temps et ont accepté que les Baruya continuent à pratiquer certains de leurs rites. Elles ont même ouvert des écoles permettant aux Baruya d'intégrer les normes fondamentales de l'économie marchande. En effet, selon l'auteur, les missionnaires éduquant les Baruya étaient également ceux qui tenaient les magasins dans la région de Wonenara. Les Baruya étaient en guerre contre la tribu rivale des Yuwarrounatché en 1983 au moment où une nouvelle génération de shamanes venait d'être intronisée (Godelier, 2017 : 120). La conjonction de ces deux phénomènes avait alimenté les peurs de la tribu Baruya. Le contact avec les colons blancs depuis les années 1960 avait en fait accéléré les processus d'évolution technique, chaque tribu essayant de prendre le dessus par rapport aux autres (Godelier, Garanger, 1973 : 188). La christianisation des Baruya est devenue plus importante en 1988 et a permis de pacifier les relations des Baruya aux autres tribus. Pour Godelier, les Baruya ont fait preuve de pragmatisme en utilisant le christianisme pour éviter les guerres et effectuer davantage de commerce. Les missionnaires blancs ont laissé peu à peu la place à de nouvelles églises locales, les haus lotu dirigées par des Baruya christianisés (Godelier, 2017 : 122). Comme les solidarités traditionnelles diminuaient, il était important, au moyen de l'éducation et de la christianisation, de pouvoir s'intégrer à l'économie de marché (Godelier, 2017 : 130). En outre, l'adoption d'une religion monothéiste permet aux Baruya d'intérioriser les principes d'un pouvoir unifié sous la catégorie d'État-nation. Religion chrétienne, capitalisme et représentation politique constituent ainsi la triade centrale de la mondialisation actuelle.

L'État-nation n'est pas une dimension contradictoire au processus de mondialisation, il est le dispositif captant localement des richesses produites à un autre endroit de la planète (Godelier, 2017 : 143). L'auteur constate simplement que les rituels liés au cannibalisme ont disparu tout comme les rites de sélection des grands guerriers (Godelier, 2017 : 159). En revanche, d'autres rôles sociaux résistent encore à l'instar du chasseur de casoar (kayareumala dans la langue des Baruya) (Lory, 1982 : 248). Le casoar représente «la femme-sauvage qui hante, solitaire, les forêts, dont la chair était interdite aux femmes et réservée aux jeunes initiés » (Godelier, 2017 : 146). On trouve aujourd'hui moins de chasseurs de casoar, mais les rituels de différenciation des sexes perdurent.

L'ouvrage s'achève sur l'analyse des catégories mondialisées de production de grands hommes à l'instar des fonctionnaires d'État, des professeurs, des entrepreneurs de l'économie 
de marché et des pasteurs (Godelier, 2017 : 157). Les Baruya se sont adaptés aux notions du capitalisme contemporain, exigeant à présent un salaire pour toute rétribution de travail (Godelier, 2017 : 147), leur évolution en quelques décennies révèle la manière dont les processus d'acculturation (Verbunt, 2011) opèrent au sein de la mondialisation créant de facto une tension entre valeurs traditionnelles et langage du business. Il y aurait un travail de traduction inverse à effectuer en prenant pour repères les catégories des Baruya pour analyser la nouvelle sédimentation sociale des sociétés occidentales et l'abstraction des rapports économiques liée à la suprématie des valeurs marchandes. Cette démarche anthropologique ne serait absolument pas originale, mais aurait le mérite d'éclairer le fonctionnement des sociétés occidentales (Abélès, 2008) qui ont provoqué le processus de mondialisation. C'est pourquoi ce serait une erreur naïve de considérer l'anthropologie comme une science sociale surannée en raison de la cartographie quasiment complète des sociétés du monde. Au contraire, la mondialisation et son cortège d'abstractions (finances, représentation politique, numérisation...) rendent encore plus impérieux le besoin d'avoir recours à l'anthropologie dans l'analyse dynamique des relations interculturelles. En outre, une réflexion sur les outils méthodologiques à utiliser aujourd'hui pour étudier l'évolution des Baruya serait précieuse pour pouvoir rendre compte de la complexité du processus d'acculturation.

\section{Bibliographie}

ABeles M. (2008), Anthropologie de la globalisation, Paris, Payot.

Ben Hounet Y. (2009), «Que faire de la tribu?», Journal des anthropologues, ${ }^{\circ} 116-117$, p. 493-515.

CAstoriadis C. (1986), Les Carrefours du Labyrinthe II, Domaines de l'Homme, Paris, Éditions du Seuil.

Drew R. (1960), Primary, Image (Richard Leacock, Albert Maysles, Donn Alan. Pennebaker, Terrence McCartney Filgate, James Lipscomb, Abott Mills), 64 minutes.

Godelier M., Garanger, J. (1973), «Outils de pierre, outils d'acier chez les Baruya de Nouvelle-Guinée », L’Homme, $\mathrm{n}^{\circ}$ 3, p. 187-220.

Godelier M. (1977), «Le concept de tribu. Crise d'un concept ou crise des fondements empiriques de l'anthropologie», Horizon, trajet marxiste en anthropologie, Paris, Maspero, p. 93-131.

Godelier M. (1982), La production des Grands hommes, Paris, Fayard.

GODELIER M. (1985), «Ethnie-tribu-nation chez les Baruya de Nouvelle-Guinée », Journal de la Société des Océanistes, $\mathrm{n}^{\circ}$ 81, p. 159-168.

GODELIER M. (1988), «Trahir le secret des hommes », Le Genre Humain, n 16-17, p. 243-265. 
GodELIER M. (2004), «À propos des concepts de tribu, ethnie et État. Formes et fonctions du pouvoir politique », in H. DAwOD, Tribus et pouvoir en terre d'Islam, Paris, Armand Colin, p. 287-304.

GODELIER M. (2009), Communauté, société, culture. Trois clefs pour comprendre les identités en conflits, Paris, CNRS.

LORY J.-L. (1982), «Les jardins Baruya », Journal d'agriculture traditionnelle et de botanique appliquée, vol. 29, $\mathrm{n}^{\circ} 3-4$, p. 247-274.

Nathan T. (1993), «Le métissage culturel : un mythe à la peau dure », Hommes et Migrations, $\mathrm{n}^{\circ} 1161$, p. $12-15$.

Piault M.-H. (2013), «Leçon 9: Anthropologie et Cinéma, Morale et Politique », Université ouverte des humanités, [En ligne], consulté le 4 juillet 2018. URL: http://www.uoh.fr/front/notice?id=2fea9221-53f6-4771-90d1-f6fa42c7a4f1.

Verbunt G. (2011), Penser et vivre l'interculturel, Lyon, Chronique sociale.

Verbunt G. (2012), «Comment l'interculturel bouscule les cultures», Les Cahiers Dynamiques, $\mathrm{n}^{\circ} 57$, p. 22-28. 